\title{
A Review on Usage Areas of Blockchain Technology in Architecture
}

\author{
Ahmet Kurnaz \\ Faculty of Engineering and Natural Sciences, Department of Architecture, \\ Istanbul Sabahattin Zaim University, Istanbul, Turkey \\ E-mail: ahmet.kurnaz@hotmail.com
}

\begin{abstract}
The development process of technology has made it possible to propose remarkable disciplines and systems that were impossible to consider a short while ago. Blockchain technology, as one of these technologies, is one of the most prominent and revolutionary technologies since the beginning of the internet to the present day. Speeding up transaction times, reducing costs, and being transparent and reliable signify the principal reasons for the transition from traditional systems to the blockchain system. Acknowledging the impact that these technologies have on various sectors, it is comprehended that blockchain technology can be used beneficially in several independent areas. So much that blockchain technology is increasing day by day, particularly in academics and the business world. Blockchain technology is presumed to influence several areas in the near future. Accordingly, this study's primary purpose is to reveal the use of blockchain technology in the field of architecture. The study was created with the fundamental idea of researching technology and understanding how it works, by being convicted that blockchain technology can solve various problems and deficiencies in architecture. This study presents an overview of the potential implementations and uses of blockchain technology in architecture. This situation has shown that we need to differentiate and evaluate differently for the business models we currently use in architecture.
\end{abstract}

Keywords: Architecture, Blockchain, Blockchain technology, Data transfer, Smart contracts

DOI: $10.7176 / J S T R / 7-04-07$

\section{Introduction}

Blockchain technology was first introduced in 2008 by Satoshi Nakamoto's article titled "Bitcoin: A Peer-to-Peer Electronic Cash System" and presented as a solution to the centralization, interoperability, and interchangeability of classical database technologies. The technology mentioned in the article has four main features. These four fundamental characteristics are as follows: The transparency of the system as well as being visible to everyone; the transactions being safe and unchangeable; the end-toend transactions, in other words, direct person-to-person transactions; decentralized, and thus managed by all persons concerned (Nakamoto, 2008). Blockchain is a database that enables value transfer within computer networks. Reliable transactions can be performed in blockchain transactions without the need for a third party. Blockchain technology has worked flawlessly for years. Consequently, it is successfully implemented and continues to be applied in various sectors around the world (Crosby et al., 2016).

With the news's influence in the media newly, various people have just become aware of the operation principles and foremost benefits of blockchain technology. Particularly in recent years, as the news imply that the personal data of users have been captured as a result of cyberattacks against some big companies and that some companies sell the user data to third parties, the expectation in the society about the need to develop alternative systems that do not depend on a central authority is progressing. Since data is not stored in a single source in blockchain systems, it is more secure than central systems. For example, if the encryption system used for the world's largest bank is hacked, access to all people's information in the system can be captured by the hacker. Since the blockchain system is not connected to a center but is created anonymously by various computers, hacking the system is much more challenging than central systems. Since hacking any independent computer in the blockchain system does not affect the whole system, it will not induce any damage to the system. The system first

61 | P a g e

www.iiste.org 
distinguishes the hacked computer as a suspect computer in this or similar case and then prevents the code verification process from being performed by this computer in case of an insecure computer. Therefore, the system's anonymous computers are frequently removed from the system if they do not give the correct verification code.

Blockchain technology, with its transparent and highly reliable structure, is a system that produces blocks by solving (mining) the passwords it produces on the computers where it is installed and intends to reward those who contribute to the block production when each block is completed. Blockchain technology provides data transfer from A to B without any intermediaries and grants several various opportunities. Blockchain technology provides significant savings in the areas used in terms of transaction costs and time.

This study strives to introduce the blockchain technology, a technology that can change the architecture sector in certain areas, and its application areas. The study describes the fundamental concepts underlying blockchain technology and the distributed structure behind the technology and concentrates on the system's potential application areas in the architectural sector. In addition, new areas emerging with the development and use of blockchain technology were additionally assessed. This study investigates how blockchain technology can influence the architecture sector, particularly in project sharing and storage. The study should be acknowledged as an introductory study, trying to introduce a theoretical framework and terminology.

\section{Purpose and scope of the study}

After Satoshi Nakamoto's article titled "Bitcoin: A Peer-to-Peer Electronic Cash System," the blockchain and the blockchain concepts elaborated accordingly have started to be studied regularly in academic and in practice. When the literature is reviewed, several studies on blockchain technology and applications are available. Despite its developing significance and awareness in other industries, the blockchain concept's scope and content have not been examined before in the architectural sector. Although blockchain technology is not practiced directly in the architectural sector, it has started to be applied in smart contracts, spending records, trade records, land registrations, construction permits, certificates, patents, copyrights, software licenses, which have common points with the architectural sector. Accordingly, it is remarked that there is a need for academic studies that systematically explore blockchain technology in architecture and review its research and usage areas.

In this study, the usability of blockchain technology and smart contracts for information sharing in a decentralized system is considered, other uses and future expectations are presented. The positive effects of blockchain technology eliminating the need for central authority on other related sectors, particularly the architectural sector, are considered. This study's principal objective is to investigate and examine blockchain technology and applications in detail in the architectural sector and make inferences and predictions about its future.

In this way, this study aims to advise those who want to evaluate blockchain technology in the architectural sector in the digital transformation process and provide support for the innovative solutions that this technology can reach and decide on its suitability. Also, it is believed that the study will be an essential resource for similar studies to be conducted subsequently.

\section{Methodology of the study}

Within this study's scope, a comprehensive international literature investigation has been conducted on blockchain technology, which is currently developing, and its usage area is becoming popular day by day. This study intends to raise awareness about the innovations that blockchain technology can bring to the field of architecture, and in this context, the fundamental concepts of blockchain technology have been considered first. In this context, information was given on blockchain technology's structure and operation, the reliability of which is indisputably at the forefront compared to classical application solutions, and its advantages and application areas were discussed. In addition, the innovations brought by blockchain technology to the sectors are declared.

To comprehend the innovations that blockchain technology can bring to the architectural sector, the blockchain technology application in various and close sectors has been reviewed. It has been examined whether the gains and innovations will create comparable benefits in the architectural sector.

\section{Literature information}

\subsection{Blockchain technology}

This section of the study contains summary information about the meaning of blockchain technology

62 | P a g e

www.iiste.org 
and its logic structure. As the name implies, the structures in which data is stored in the blockchain system are called blocks. These block structures are arranged in a chain (a linear array in time) called a "blockchain." Blockchain technology, which came to the agenda with the article on Bitcoin published in 2008, can be described generally as a system where every user entering the system without the need for any intermediary can share all kinds of information at the same time, meet and exchange within the context of supply-demand. The technology in question also enables the transfer of valuable and confidential assets in the existing internet environment that provides data transfer (Url-1). The blockchain concept is essentially based on 'Decentralized Distributed Ledger Technology' (Altunbasak, 2018). Blockchain is a decentralized database where all data and transactions between participants are stored in a distributed manner. Each stored transaction was recorded by agreeing with the majority of the participants in the system. The accepted transactions are added to the blocks list and stored. The blockchain continues to develop with the addition of new blocks. The blockchain comprises an accurate and verifiable record of every transaction made.

When recording new information to a block, previous blocks' information cannot be edited or changed. Linking the contents of the block newly added to the blockchain to the previous block is performed using cryptography (encryption). To guarantee account consistency and user security, blockchain technology ensures its continuity by using asymmetric cryptography and distributed consensus algorithms. Any unapproved change in a previous block's content in the chain invalidates the data in all blocks. Distributed consensus logic requires that the transaction be confirmed by most computers within the system to make a transaction on the system. The transaction, whose verification has been completed, is recorded, and it is not possible to amend or delete the transaction from now on. Blockchain is consensus-oriented. Many computers are connected to the network. To overcome an attacker's ability to make transactions on the network maliciously, those added to the blockchain must strive to solve a mathematical challenge. Results are shared with all other computers on the network. Computers or nodes connected to this network must agree on the solution. Consequently, the expression of consensus is a characteristic that stands out in this technology. In other words, no single entity can take control of the information on the blockchain. The advantage of this structure is that transactions recorded on the blockchain can be publicly published and verified. Anyone can view the blockchain content and confirm that the recorded events have occurred (Linuma, 2018).

In simple terms, a blockchain is represented as a list of records shared by stakeholders within the network's boundaries, signed in cryptographic form, which cannot be altered after the transaction is made. All of the maintained records incorporate a time reference linked to previously maintained records. The most significant point that makes the system secure and transparent is that no retroactive changes can be performed, and the transaction records can be observed by everyone participating in the system. In this system, unlike known databases, there is no particular authority; transactions can be carried out autonomously between individuals and observed by all participants (Turhan, 2018).

\subsection{Smart contracts}

Thanks to blockchain technology, contracts can become smart with the control process on anonymous computers. Smart contracts are a piece of code on the blockchain. The system turns the promises between the parties into a contract as a way of working. The contract is realized automatically when the requirements are fulfilled. If one of the parties does not fulfill one of the conditions, the system cancels the contract.

If we think of the blockchain as a distributed database, we can comprehend smart contracts as applications that can perform transactions with this data. Although not directly linked to contracts in the legal sense, these programs, called smart contracts, consist of program commands that will perform the transactions they have previously agreed on the accounts included in the contract. Once smart contracts are involved in the blockchain, they cannot be altered. The part they can play in process automation is enormous, as they contain commands that can be processed automatically by the machines involved in the blockchain when the terms of the contract are fulfilled (Buterin, 2014).

For example, in the assumption that medicine sale contract in a truck to be transported from Germany to France is signed through smart contracts, if the following statements are included in the contract; "If the drugs are not delivered within 15 days, the contract will be terminated." and if the delivery is not realized within 15 days, the contract is terminated without requiring any intervention. Or in this contract, if a provision implying a reduction of 2000 Euro for each day delayed after ten days is included, the contract amount will be reduced by 2000 Euro for each day the contract is automatically delayed, assuming that the goods are not delivered at the end of the 10th day. It is predicted that the

63 | $P$ a g e

www.iiste.org 
agreements concluded in this way will create a radical change in the whole world as they can be performed and registered securely (Savelyev, 2017). Another example is a smart contract that includes commands for renting a vehicle that operates with a smart lock; the contract parties are the person who wants to rent the vehicle and the account that owns the smart lock. When the agreement is reached, the rental fee is transferred from the account of the person who wants to rent the vehicle to the contract, and the smart lock is turned on. When the contract expires, the amount previously transferred to the contract is transferred to the account that owns the smart lock, and the lock is turned off (Buterin, 2014).

\subsection{Advantages of blockchain technology}

Blockchain technology reduces the need for a central server or a trusted authority to transfer value. Instead, copies of the data are stored on thousands of computers working together, and amendments to the data are achieved by agreement between nodes. In blockchain technology, every modification that happens to a data is recorded with a timestamp to ensure transparency. In the system, the confidence between stakeholders who do not need to identify each other is guaranteed by ensuring that amendments on the stored data can be performed following the determined rules, and by keeping copies of this record chain with the parties by maintaining these amendments in a transparent record chain, protected by cryptographic techniques.

The main advantages of the blockchain system are as following: the lack of a central unit (distributed database) in the blockchain, the reliability of the system, the rapid performance of transactions, the ability to monitor the system where transparency is at the forefront effortlessly, the inability to change the transactions, the presence of user-controlled networks, controllability and low transaction costs (Demirel, 2017).

Distributed Database: A blockchain is a distributed form of registration that facilitates recording transactions and tracking assets on a network. Every participant in the blockchain has access to the whole database and history. So, one party does not control the data. Each participant can directly confirm the transaction partners' records without the need for an intermediary. Blockchain technology enables these systems to operate more efficiently by developing the fundamental trust structure in systems based on centralization, which is regarded as a modern problem to a vast user network. All contributors have the same rights to blockchains. This means that anyone can view the blockchain's content, make transactions, and contribute to the security and integrity of the blockchain.

Security: In the digital order, which brings potential threats to personal information and the convenience and advantages it brings to human life, the secure storage, transfer, and protection of data against possible danger has become an issue that brings both governments and private organizations' attention. In blockchain technology, transaction records are stored consecutively in blocks. Successive blocks of saved transaction records are also successively added to the current blockchain. Consequently, it is greatly difficult to counterfeit. It is impossible to change the records retrospectively after a record is created in the distributed books in the system or after they are spread in the network. Since each block is linked to the block that preceded it, the block's hash value is modified with a change to be made within a block. Since the chain's integrity will be disturbed due to the change, the block in which the change is experienced is not addressed by the following block. In such a case, the chain's integrity will be lost, and users in the network will not accept the change (Mendi and Cabuk, 2018).

Fast Processing Times: The blockchain system developed has a structure that is suitable for constant operation 24 hours a day, 7 days a week. In this case, transactions can occur more quickly, without interruption, through the blockchain base.

Transparency: In order for the concept of transparency to become valid, all users involved in the system can view the blocks within the scope of blockchain technology and all the transactions within the blocks (Yli-Huumo et al., 2016). Hence, the system becomes much more transparent than systems in which a central unit is located in the managerial position.

Failure to Modify Transactions: Records cannot be amended after a transaction is entered into the database, as any unauthorized change in the content of a block in the blockchain nullifies the data in all blocks.

User Controlled Networks: The development of a system that users can control instead of centralizing the network is an essential advantage. Rather than establishing a central party in the system to process data, stakeholders control each other within the system and determine how to act in the continuation of the process (Demirel, 2017).

64 | P a g e

www.iiste.org 
Controllability: Since the system stores all transactions as a part of the chain, all transactions can be followed and monitored. In this way, even 100 years from now, it is reasonable to monitor a simple data transfer realized today. This allows to track and follow the chronology of transactions.

Low costs: Blockchain-based systems can be developed at much lower costs than current systems with similar security levels by connecting technology elements such as distributed structure and consensus protocol (METI, 2017).

In line with all the advantages listed above, it is predicted that the practical application of blockchain technology can create radical changes in various areas and fields and may lead to the emergence of very significant innovations.

\subsection{Structure and operation of blockchain technology}

The blockchain technology's operation process can be briefly summarized as follows: When a user wants to transfer or update any data on the blockchain, the data is primarily published to all nodes in the blockchain network. Each computer in the blockchain system is called a node. New data is turned into a block by the nodes and presented to other computers' control and approval in the blockchain system. Computers in the blockchain system called this node perform several mathematical operations to control the data block's security and control its compliance with system protocols. If most computers in the system approve the block, the encryption phase is initiated as the next phase. At this stage, the data block is encrypted with a password, and each encrypted block includes a password belonging to the previous block. Hence, while the blocks are encrypted, they are not merely encrypted but also linked to each other with the formerly encrypted block (Figure 1). The reason why the system is called blockchain is precisely because of this operating principle. Ultimately, other computers in the system approve the new data block encrypted and added to the previous encrypted block and thus added to the blockchain system not to be modified or deleted (Gunen, 2018). Millions of computers carry out all of these operations in the blockchain structure, and the people who complete this encryption process are called data miners; and this process itself is called data mining. In return for the service in question, the system rewards the users who help the security service and even become a part of the system, creating a source of motivation for them.

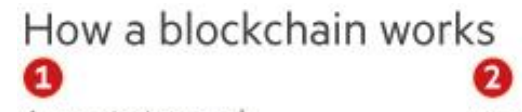

A wants to send data to B
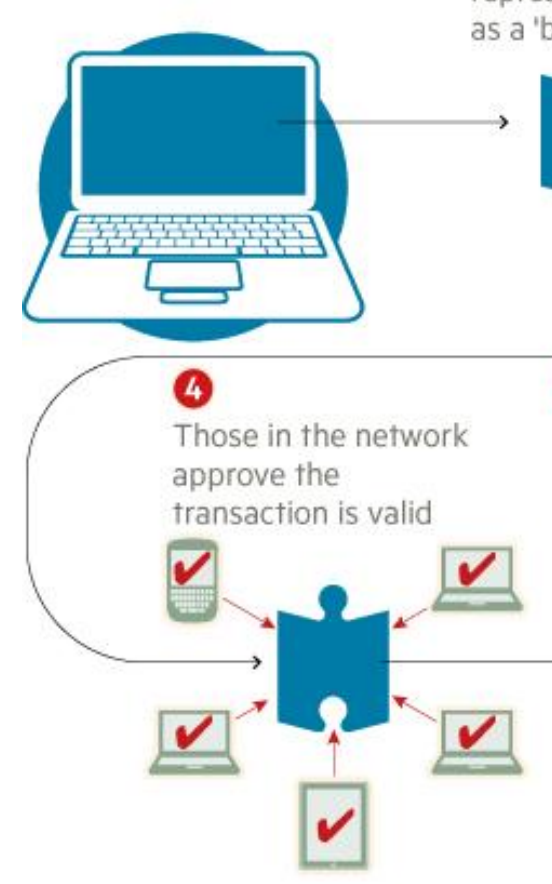

The transaction is represented online as a 'block'

Figure 1. How a Blockchain Works (Wild et al., 2015)

(3) The block is broadcast to every party in the network ? ?

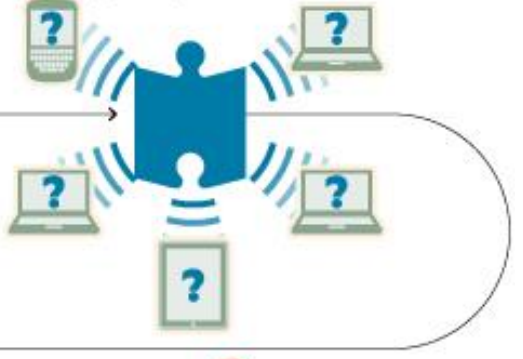

6

The data moves from $A$ to $B$ to the chain, which provides an indelible and transparent record of transactions

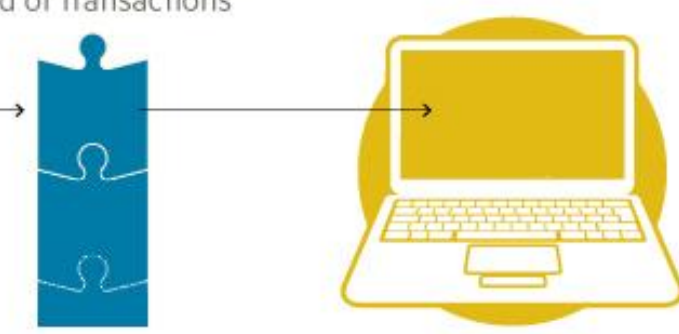

65 I P a g e

www.iiste.org 
Baran (1962) refers to centralized, decentralized and distributed structures in his article. In centralized systems, information is kept in a single-center, and all authorities, such as changing and deleting information, belong to this center. This situation enables the system to be managed from a single point of control, and a severe problem in the center can disrupt the entire system (single point of failure). The decentralized structure is formed by the connection of more than one atomic central structure, and there is no single center for the whole system. The problem of decentralized structures is that destroying a certain number of units can stop communication within the system. Distributed structures are essentially similar to decentralized structures. Nevertheless, these are structures where units that do not have the central system characteristic, even in the smallest unit, the structure can communicate with other units directly or through other units. In such systems, control and risk are distributed among the units in the network. All computers' coordination in the system in central structures is performed from a center, and data exchange takes place through this center. In distributed structures, all computers are in equal positions. They all contribute and use the system (Baran, 1962).

The blockchain is a digital record where users can view information on the network, and anyone can use it. It has a structure that can be operated not by a person or organization, but by thousands of processors located in various parts of the world in a distributed network. Blockchain technology is a distributed database providing encrypted transaction tracking. The difference between a distributed network structure and centralized or decentralized network structures is presented (Figure 2). Each point in the network types shown is designed as a computer. Any rule or authority does not restrict computers in a distributed network structure and the data they transmit.

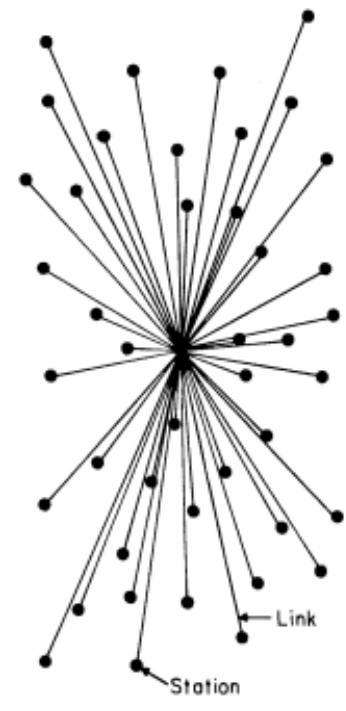

CENTRALIZED

(A)

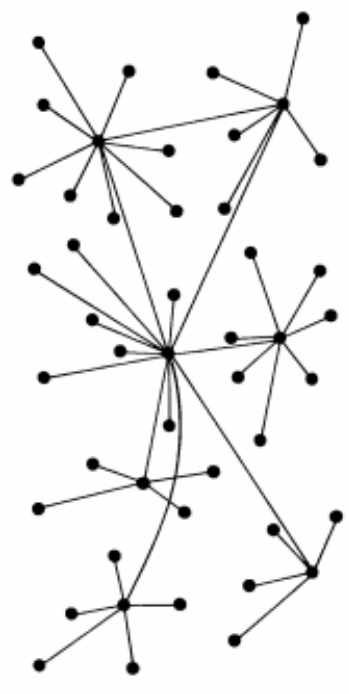

DECENTRALIZED

(B)

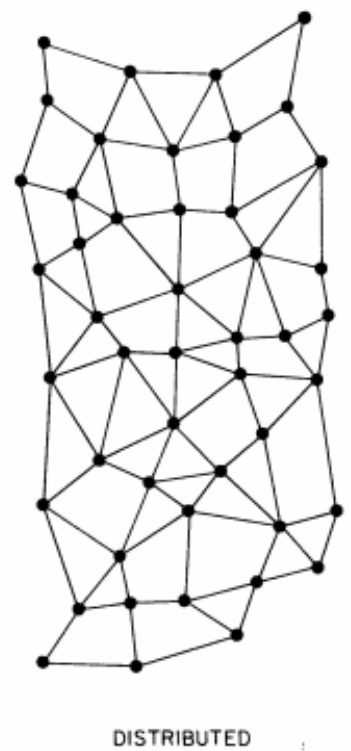

(C)

Figure 2. Network Structures (Baran, 1962)

A distributed database can be explained as the record of each of the transactions on the system's computers. The data in the system are saved in conjunction with all computers in an open network. If this condition is met, there is no need to establish a central unit, so traditional systems' risks and costs are not confronted. This new database system was developed for the first time in the early 2000 s and implemented through networks such as eDonkey and BitTorrent (other people you do not know on the internet, but you can communicate with, more precisely, a kind of data storage and sharing developed for peer to peer (P2P) developed for sharing data over computers and software), etc. Nevertheless, several risks have arisen, such as changing the existing contents, because the contents are not given a password within the structures mentioned above, and choices are not offered for the places where the data will be stored. Common ground is created within the scope of the developed blockchain technology, in the entire network, to provide a conjugation to solve the existing problem. When the system is generally evaluated, it refers to establishing a data storage system where multiple parties meet based on a preliminary agreement, eliminate errors, use other databases in recording transactions, and avoid changing transactions.

66 | P a g e

www.iiste.org 
Blockchain applications save and protect digitally obtained data using cryptography. Using cryptography in the system in question, the users are provided with the possibilities of high-reliability blockchain technology, which can be executed simultaneously over several points without any control center, which is highly reliable. In this case, the data is transferred to the stakeholders within the existing authorization scheme, and thus it is guaranteed that the transactions completed are accurate and consistent. In other words, in order for any transaction to be performed, simultaneous approval must be received from the stakeholders active in the system. Since the central type of transaction is not included in the structure, transactions completed are recorded by other stakeholders, and in parallel with this situation, each transaction is stored based on the previous transaction. In this case, the principal benefit of the emerging new blockchain technology is that the trust problem between stakeholders can be eliminated without the need for high-cost security systems. This means a significant increase in productivity for stakeholders.

The distributed database, where each information is stored in blocks with advanced encryption algorithms, enables us to perform transactions without being linked to a center. The most prominent characteristic of this system is that it is saved in more than one place, i.e., in a whole network, instead of being kept at a single point. The reason for maintaining records in more than one place is to increase the reliability of saved information. Even if one or more of the storage locations are lost, information continues to be stored in other storage locations on the network. The places where the information is saved are concerning the previous block and next block with unique encryption. In this case, when information is modified in one of the rings that make up the chain, this information becomes incompatible with the previous records (Egilmez, 2013). In blockchain technology's operation process, each block contains the previously created block information, and thus the blocks become a chain by interlocking (Carkacioglu, 2016). Modifying any of these interconnected blocks makes it impossible to attack. In other words, it prevents malicious attacks from outside. Because when each block is discovered, proof of work, in other words, the previous block's content is demanded. A malicious end that wants to modify any block will have to modify all the subsequent blocks. Nevertheless, since proof-of-work is required while creating each block, an end attempting to change blocks will have to create a proof-of-work in all blocks forward. This is not possible as it will require enormous processing power. This characteristic is one of the most significant powers of the blockchain (Carkacioglu, 2016). Blockchain contains approved and interconnected blocks. It is updated continuously to ensure the integrity of the information and is distributed to all participants. Any party joining the network will obtain the latest version of a blockchain file (Pinna and Ruttenberg, 2016). If the blocks are considered the pages of a book, the blockchain can also be thought of as a book. Structurally, a blockchain is a set of securely chained blocks of information. New blocks are determined when participants generate new pieces of information. After the creation of the first block, every subsequent valid block must contain the hash output of the previous block. Thus, a piece of reliable audit evidence is created by ensuring the blocks' security (HKMA, 2016). The principal motivation behind these transactions is to create an architecture of trust. The blockchain structure enables participants to share the updated records through peer-to-peer replication every time a transaction takes place. The peer-to-peer notification indicates that every participant (node) in the network acts as a broadcaster and a subscriber. Each node can send or receive transactions to other nodes. Data is synchronized over the network since the moment it is transferred (Gupta, 2017).

\subsection{Usage areas of blockchain technology}

The 2008 global crisis caused consumers to lose confidence in the banks, financial, and central supervisory institutions. In a crisis environment, for the first time, the concept of blockchain emerged as a solution that includes precautions against manipulation, that lives without the need for the ownership of authority, can work without being connected to any central system, and that all users can use it without any confidence problem. It developed quickly and turned into a globally trusted technological concept. It became so popular that blockchain technology was compared with the revolutions of the press, steam machines, and the internet. Blockchain technology expands its ecosystem with new application areas and new technology components and is being adopted by a growing audience. It has been remarked that blockchain technology eliminates intermediation in many sectors and allows less costly commercial and individual activities with a reliable computer system.

The blockchain technology focused on in this study creates innovations that can be integrated with the business world. Blockchain technology, which has a distributed structure in terms of network, stands out as one of the most powerful digital revolution channels. Personal data resulting from human life's

67 I $P$ a g e

www.iiste.org 
digitalization has become one of the most valuable concepts of our age. With the increasing data traffic, these data's storage, retention, and transfer processes have become essential. The protection of personal data is one of the most discussed topics in recent years. Today, data transfer is performed in several areas (multimedia, communication, social media, construction, etc.). Blockchain technology, on the other hand, is a distributed database that enables us to transfer assets that we attach value to besides this data. It has been acknowledged that blockchain technology may be used more generally. Blockchain technology has a much more comprehensive range with the possibilities and various applications it provides (Url-1).

Blockchain technology is a valuable technology that works as digital record storage and can be applied to different areas (Dilek, 2018). Blockchain technology has attracted many diverse industries due to its decentralized structure and special security measures. Blockchain technology is regarded among the technologies that can cause many massive transformations comparable to artificial intelligence and the internet of things technologies (Unsal and Kocaoglu, 2018). Since this technology's security measures are more productive than existing network structures, it has been applied to many areas that demand reliable data transfer. It is possible to comprehend these vital technology's application areas in several areas, particularly in the financial and public areas (Unsal and Kocaoglu, 2018). With blockchain technology, all kinds of structural information can be registered, regardless of the party making the payment. Consequently, blockchain is a technological development that can operate in many diverse sectors, such as logistics, retail, supply chain, energy, e-commerce, real estate, and health. Also, it is possible to observe the blockchain technology in many areas, including the registration of real estate, vehicles, and valuable assets; maintaining birth, marriage, and death documents, digital passport, social security system, tax system; conducting elections, organizing surveys, keeping accident or criminal history about the vehicles, notary procedures, land, and house deed records, and management of the smart contracts (Unsal and Kocaoglu, 2018).

Finance: William Dudley (President of the New York Branch of the Federal Reserve Bank) highlights the significance of using blockchain technology in finance. Blockchain technology is used in the financial sector, payment transactions, money transfers, buying/selling platforms, clearing management, authorization, verification, digital identity management, document management, etc.

In recent years, the concept of Bitcoin and crypto money has started to be among the topics that have become extensively examined in various sectors and fields such as finance, particularly in the media. Bitcoin is the first cryptocurrency. It is essential to know the blockchain technology and infrastructure behind it to comprehend Bitcoin, which is considered virtual money. Contrary to popular belief, blockchain and bitcoin are separate concepts. Blockchain is the technology used in the bitcoin system and has numerous other uses. Because the Bitcoin network utilizes blockchain technology, it has a decentralized and end-to-end structure. It does not have a central server or control point. The Bitcoin network structure is different from traditional money transfer tools. Traditional money transfer systems (such as banks, brokerage houses, VISA, PayPal) are usually centralized (Carkacioglu, 2016). The Bitpay platform, improved using blockchain technology, is a payment tool that allows virtual or real companies to receive Bitcoin payments (Url-2).

Blockchain technology is generally recognized as the technology behind virtual currencies such as Bitcoin and Ethereum. Ethereum's founders refer to Bitcoin as the first generation and Ethereum as the second generation. With the support of companies such as Intel, AMD, Microsoft, etc., an association called Enterprise Ethereum Alliance has been established to structure a specific blockchain to make it available to the corporate world. This Ethereum-based association has become the network system supported by several more companies in the future. During this process, Microsoft also focused on Ethereum technology with its cloud-based enterprise information system called Azure. It created a design in which cloud-based blockage transactions, such as corporate accounting systems, can be carried out efficiently and offer this service to its customers by making international payments and trading transactions. Several insurance companies and bank institutions are currently utilizing the Azure platform (Microsoft Cloud Computing Services).

It may take a few days for transactions, such as money transfers, to be completed in the banking system. The principal reason for this situation's emergence is that financial institutions work within working hours, five days a week, and together with transfer software protocols of the banks. Also, due to the difference in local times in various parts of the world, transactions can sometimes take weeks to complete. (Demirel, 2017). Currently, several financial institutions have begun testing transactions in blockchain technology. Standard Chartered utilized Ripple, an enterprise-level blockchain application, to perform its first international transactions. For example, the Ripple platform has currently completed

68 I P a g e

www.iiste.org 
a clearing process performed in 2 days in the regular banking system network in 10 seconds. An employee of the National Bank of Australia utilized Ripple's ledger technology to transfer \$10 from his account to another employee at the Royal Canadian Commercial Bank. This process took only 10 seconds (Guo and Liang, 2016). Blockchain technology has broken new ground by producing more reliable, faster, and less costly transactions than many traditional banking products and services.

Data Storage and Transfer: Today, storing and transferring data on the internet with cloud technology has become the most used method. These individual users are provided with applications such as iCloud, Google Drive, Dropbox, and companies. In a centralized structure, cloud storage is vulnerable to risk factors and security threats, although it has an important working principle. Considering this, instead of being stored centrally, using the distributed data system provided by blockchain technology, this technology will provide users with benefits such as healthily storing information, preventing it from being lost, and restricting it from being hacked. On the other hand, users in the blockchain rely much more on cloud storage, as they are in a decentralized system that can prevent data loss. Some alternative blockchain-based cloud storage projects to central cloud data storage services being developed in this direction can be listed as Filecoin, Maidsafe, Storj, and Siacoin. For example, Storj and FileCoin enable people who have a memory that they do not use on their computers to rent these free spaces and those who need memory to store their files on the network securely (Wilkinson et al., 2014). Likewise, blockchain-based alternatives to many services currently offered on cloud platforms are emerging.

Copyright: The Internet has made access to information very easy today. All people in the world have gained the ability to receive, copy, and even spread the information they want whenever they want. Although this information access is a good achievement, it has led to the development of copyright problems. Once a logo designer has designed the logo, the designer has just one customer, sometimes not, to sell it while protecting copyrights. Blockchain-based applications have been created for solving problems such as protecting intellectual property rights that commercial companies have not solved for years. With smart contracts concluded with blockchain technology, the creator will deliver their rights directly to the sellers and retain their rights through the system.

The application Mycelia, which British musician Imogen Heap established for artists, is used for vocal artists and writers. In this way, songwriters can sell their lyrics directly to studios and retain their copyrights. Mycelia is a blockchain project that enables artists to store all the information about their songs in one place and track payments more efficiently. Additionally, Mycelia enables music professionals to hold a searchable database and systematically communicate thanks to blockchain technology. In this system, the blockchain application can be used actively and smoothly. It is considered that the infrastructure that will give the most reliable and secure intellectual property rights will soon become a blockchain. In this context, it is possible to prevent some art difficulties, such as not sharing, being stolen, or misrepresented. Spotify is also expanding its work in this direction. It acquired the blockchain company MediaChain, which was built to regulate better the royalties distributed to artists.

Content Publication: Steemit is a blockchain-based content publishing platform that serves publishers as a field of application and where readers reward qualified content. The platform currently has close to 1 million subscribers, and more than 1.5 million content is published on the platform each month. The Steemit platform has its own blockchain infrastructure. It is also stated that the platform can protect copyrights as it becomes a record that cannot be destroyed with the publication of content on its blockchain platform.

Real estate: For the real estate sector, prices can be defined and secured by land or property owners through smart contracts. Some of the projects generated using blockchain technology in this direction can be listed as ShelterZoom and Ubitquity.

Supply Chain: By minimizing the margin of error in international trade and logistics, which are significant for the supply chain, more prosperous commercial activities can be carried out with less costly and higher quality process management thanks to blockchain technology.

Tourism: In the tourism sector, which is one of the principal income items of many countries, studies are carried out on blockchain technology to provide solutions to the difficulties experienced in loyalty programs and online consumer comments.

Game: Today, the game industry is under the control of companies that make large investments. With the blockchain technology, small-scale game companies have the opportunity to expand their games all over the world without being subject to permissions or fee problems. It is possible to give the Bountie project as an example in this area.

$69 \mid \mathrm{P}$ a g e

www.iiste.org 
Donation: It is possible to make donations transparently through blockchain applications. Because it is easy to track where the donations made within the system go. Giventh and Alice developed in this area are among the significant projects.

Education: With another developed draft blockchain application, universities can keep their graduates' diplomas in the blockchain in a non-changeable, retrospective, and transparent way. Employers can inquire about the validity of the diplomas offered by graduate students. The latter applies for a job on this blockchain. Since the diploma data are not stored in a central database but are recorded in the blockchain, it does not allow any intervention. Thanks to this application, unfair gains and diploma fraud can be prevented.

Health: In the health sector, patient information cannot be modified, deleted. Simultaneously, a health history file that can be used worldwide can be given by blockchain technology by securely storing it so that it cannot be seen outside the patient's initiative.

Blockchain enables the safe and automatic transfer of an asset without the need for any intermediary, as in the application areas specified above. Although blockchain technology has been interpreted and evaluated primarily in financial technologies, it seems that there will be numerous other applications. New ones are continually being added to the application areas that are currently being studied. Today, many institutions continue their R\&D processes and pilot studies on reconstructed usage scenarios. According to a large-scale report by Switzerland-based Credit Suisse, blockchain is utilized in several areas, not just financial services. According to a survey conducted with 800 executives by the World Economic Forum, 58\% estimate that $10 \%$ of the global Gross Domestic Product will be stored on the blockchain before 2025. Although thousands of services develop solutions using existing blockchain platforms today, investments in applications belonging to blockchain platforms continue to increase day by day. In addition to these applications, blockchain technology seems possible to be the basis of several applications and industries that we cannot predict in the future (Xu et al., 2017).

\section{Blockchain technology in architecture}

Blockchain technology can be defined as a technology that enables data transfer between computers in the peer-to-peer network transparently and reliably. Communication in peer-to-peer networks, data storage, and encryption methods in distributed systems are not new computer science topics when analyzed separately. Blockchain technology, which emerged from their combination, made many innovative applications achievable. The process of hosting all kinds of digital data can theoretically be applied to blockchain technology. Since it is a database system without a central authority, it cannot be changed due to its distributed structure. Since retrospective corrections cannot be made, it is possible to use the blockchain technology in many areas. This study intended to reveal the potential application areas of blockchain and smart contracts in the architectural sector in the context of the specified transparency and reliability principles.

With its transparent structure, user-controlled network, fast transaction execution, low cost, unchangeable information, distributed data network system, high-level encryption methods and security approach, and confidentiality, the blockchain technology can provide solutions to creative sectors' problems such as architecture and design, and it can improve the current situation. Simultaneously, its integration with technological developments such as the next-generation internet and the internet of things is significant in security. The use of blockchain technology and smart contracts in architecture can lead to significant developments in the regulation copyright, in increasing project and construction efficiency, collecting documents, making secure payments (service fees, progress payments, etc.), avoiding time losses with fast data sharing, crowdfunding for projects, project sharing system, digital records. Possible applications and areas where blockchain technology can be easily integrated with the architecture and offer future-oriented solutions are specified in the study. The primary possible applications and areas where blockchain technology can be used in architecture are defined as follows in the study; data transfer and storage, payment, storage and publication of architect and student projects, processor sharing and copyright issues. The study strives to define application structures and working methods.

Data Transfer and Storage: For architectural projects, blockchain networked data transfer and storage can be classified as the first of these potential areas. Particularly, with the beginning of the 21 st century, thanks to Web 2.0 technology, which rapidly digitalized the whole world, total data volume combined in thousands of years has accumulated in just a few years. As a result of this sudden and fast digitization, the data was immediately transferred to the computer environment. This situation has led to an unprecedented data density. As a result of this extraordinary density, some new requirements have

70 | P a g e

www.iiste.org 
emerged, such as the storage, classification, and safe transfer of the enormous data stack when required. There is a multi-stakeholder system structure in the architectural sector. In this regard, blockchain technology provides security, transparency and cooperation solutions for data transfer and storage in architecture. In the architectural design processes generated using blockchain technology, data transmission to all stakeholders concurrently through a distributed data network system provides users with reliability, convenience, and time advantages. Making information accessible to all network users can reduce errors and be stored in a blockchain-based cloud environment, where accessibility can be guaranteed. In particular, thanks to blockchain technology, stakeholders can access related information and projects and gain transparency by making their management practical. Data transfer, accessibility and transparency in architectural processes act in direct proportion to efficiency in all transactions.

With blockchain technology in architecture, data records that are continually developing can be distributed to users without modification. Since data in the blockchain system is backed up on several nodes, it is also possible to save only the hash of a given data set. This can also be considered as a unique fingerprint of the data. Thus, the blockchain's size becomes controllable, as the size of the hashes is much smaller than the size of saving the actual data. Since the data is stored in a distributed manner over communication networks, it ensures a consensus that the data remains related at all points during this process. Also, since all data will be encrypted, the user will use the data alone. If the user gives permission, other parties will be able to access this data. Blockchain technology can make a universal contribution to the architectural sector in terms of storing, classifying data and transferring it securely when necessary.

Payment: Delay of payments is a frequent problem that negatively affects all projects and constructions in the architectural sector. Late payments cause significant problems in architectural offices' cash flows and construction site management and personnel payments. Despite the importance it offers, little researches or practices have focused on growing systems that will guarantee participants to pay on time throughout the project and construction. Recent developments in blockchain technology and smart contracts can provide a secure platform for timely and guaranteed payments on architectural projects. Smart contract-based payment systems hold significant potential for both payment problems and trust between parties. This study recommends to design and develop smart contract-based systems for a timely and guaranteed payment of architectural projects. Smart contracts should be developed for a guaranteed payment of project cost and progress payments. In this way, it can be intended to accelerate the current project price and progress payment system and to reduce the disputes that may arise due to the payment. Blockchain technology can be a solution to one of the most significant architectural sector problems at this point.

Storage and Publication of Architect and Student Projects: Generally, projects designed by students who receive design-oriented education, such as architecture and interior architecture, are created during their education period and are archived in their computer or external memory after receiving the relevant project's grade. The same is valid for architectural firms and architects working as freelancers. If the project's application is not admitted or if the project is created for other reasons will not be used again; these projects will remain in the company's archives or personal archives. Nevertheless, if all these projects are announced via a platform instead of archiving, others can benefit from these projects, and even the people or organizations that create the projects can earn income from these projects. If we consider the innovative ideas in this field, a blockchain-based platform can be generated where people and companies in the architectural sector can share their designs and projects. On this platform, persons and institutions connected to the network can save their designs and projects with their knowledge and skills, process their architectural contact information and publish them in the system, transfer them directly to others in a particular way, sell all or a part of their projects for a certain fee. They can take it securely over the blockchain and comprehend how much money all your designs and projects earn transparently on the system. On the other hand, participants on the network can find people who have the knowledge and skills required for their job descriptions, buy the design and project they need, invest in the stakeholders' development, and then become a partner with the earnings that people earn from them.

The metadata embedded in each architectural project saved on this platform, which can be created with blockchain technology and smart contracts, can include the terms of use and contact information of the project owners, as well as making it much easier to locate the registered project owners and obtain the required permissions to use. These projects can be stored on the blockchain using the cryptographic hash method. The blockchain technology's distributed database structure will provide immediate and automatic updating of the system's data. With the blockchain platform that can be developed, issues

71 | $\mathrm{P}$ a g e

www.iiste.org 
such as participant payments or copyright can be realized transparently with the fair and quick sharing of the participants' income. The whole architecture database can be transferred to this system. This system can encrypt every architect's digital data at a very high level and process it into the database. In other words, this platform can be considered as a shared architectural project library, contributed by every generation. Each participant can add one or more blocks to the chain, allowing the block to expand. The more blocks that the participant adds, the more blocks are available in the chain. The shareholders who process blocks in the chain can be rewarded as a reward in proportion to the blocks they have committed, or according to the blocks' usage rates they have processed by other participants. Thanks to this platform, KYC (Know Your Customer), the architecture sector will reach various in the blockchain system. Since the comments presented for the design or the project can be recorded on the system in an unchangeable form, other participants can find the opportunity to purchase by being certain of all the project's characteristics or design to be purchased. In this way, transparency will rise in the entire system, and the problems can be eliminated. Thanks to KYC systems, what the parties need to trust each other can be acquired safely and quickly from old records. With this application, which is used for banks to provide loan support to their customers, it will be possible to transparently see the parties' previous actions in the architectural sector. With blockchain technology in the sharing economy, the parties will not have to pay the commission fees. Direct action from person to person will be possible. Thanks to P2P payments between users, payment transactions can be carried out promptly and securely without the need for an intermediary. In this way, the commission fees paid to the companies will become an expense item that the parties do not have to endure.

Thanks to this application's P2P system, sharers can broadcast data to millions of people simultaneously without having to send a project to people individually. Project data can be located not in a single-center, but distributed over millions of computers. Some of these computers may contain all of the data, and some may partially contain a certain part of the data. When access to any data is requested, the system directs the person to get this data from several computers with the most optimum arrangement possible. This application offers a solution exceeding current cloud technology.

Work should be performed on such a blockchain structure that can store millions of project files from various individuals and institutions, publish them to all users, and contain information about who should pay whom and how much and how to contact whom. As a result of these studies and the placement of architectural project data into the blockchain system over time, a very comprehensive and extensive database will be created on behalf of the architectural sector. These systems, which do not have the risk of being shut down, being hacked, being stolen, or shared without the user's consent, are likely to become popular over time, where prices are more reasonable.

Copyright: Keeping the copyrights of digital architectural records on a blockchain network will help understand this situation if the record is examined and copied using reconciliation systems. Consequently, the security of copyrighted data in the architectural digital world will be guaranteed. The reconciliation to be completed on a digital system must be guaranteed by software. At this point, the blockchain system comes into play. The blockchain system can be described as a system that guarantees that any copyright information in the digital environment remains the same at all points in a distributed manner (open to all users) over communication networks (Sahin, 2018).

Processor Sharing: Blockchain technology can also be used for architectural rendering and animation applications. The processor load required for the renderings and animations can be distributed to the people who are connected to the system and do not use the computer processor, by adapting the rendering and animation implementations that require the high processor to this system. Individuals and institutions that need rendering can have transactions requiring a lot of processing power by paying less money than they normally pay. Participants who contribute to rendering and sharing their computer processing power can earn as much income as each data they process. With this kind of application using blockchain technology, a computer's work can be distributed to all computers on the network and completed much faster. Thus, processes that require the long-term, uninterrupted operation or a high processing load can be allocated to normal computers instead of a supercomputer in proportion to their processors' power.

\section{Conclusion}

In recent years, technology and the innovation it brings have an impact on many fields. Blockchain technology appears to be a powerful development, with what it offers and promises for the future. The fact that it can be applied to almost every field provides serious benefits to both consumers and companies. States, companies, and individuals need to follow the developments in technology today,

72 | $\mathrm{P}$ a g e

www.iiste.org 
where the concept of technology exists in every field worldwide and can directly affect essential points. Recently, blockchain technology, which attracts the attention of the whole world and provokes curiosity, is a concept that is becoming increasingly popular.

Although blockchain technology is perceived as a technology that allows only data transfer, it has revolutionary technological infrastructures that can potentially be applied to almost any industry. Intermediary and safe value transfer can be announced as the starting point. It installs the blockchain intermediary function on a transparent and highly secure system. It can be said that it acts as an honest arbitrator, loyal to the terms agreed on by all parties in commercial or individual contracts. Blockchain technology is recognized as the entry of new data of many participants in a limited or undefined number of peer-to-peer networks, the validation of new data blocks, and the addition of these data blocks (previously verified data) to the chain. In other words, with the blockchain created by adding each block to each other, each transaction is recorded and can never be deleted. The algorithms achieved with this method become closed to intervention not to be reversed by the human hand (Altunbasak, 2018).

Blockchain and extension tools can be used in architecture, as they can be used in many sectors. With the increasing use of blockchain technology in the architectural sector, the data may analyze the industry's future. Blockchain technology can enable the use of a distinct functioning and structure that is not yet foreseeable but may affect the use of existing established architectural relationships and systems. Society, particularly architects, can effortlessly benefit from it.

The multi-disciplinary interaction between architecture and technology requires powerful collaborations between architects, users, and regulatory authorities. Academic studies on blockchain technology planned in architecture will increase awareness in this field and improve literacy about the future of architecture. Academic reviews to be conducted in terms of governments and international policies will make their legal status more apparent. Distributed systems and blockchain technology are included in the academic programs of the world's leading universities. The inclusion of blockchain technology in academic programs will benefit developing and transferring information and technology and fulfilling the demand. When we consider all these benefits, it is predicted that blockchain technology for the architectural sector will bring innovations that can alter the foundations in many different areas.

In the study, implications were made to identify the opportunities in blockchain technology developing all over the world, analyze the effects of these opportunities on architecture, and determine innovative strategies. In the coming years, architectural data can be moved on this system. Individuals and companies can keep their archives on this system. The most significant characteristic that distinguishes blockchain technology from other technologies is working together in environments such as crossindustry consortiums and platforms. As a new mindset, the blockchain enhances ecosystems' significance. It highlights ecosystems that can create value together rather than individual companies and their products. For this reason, the public, private organizations, and academicians should collaborate to start studies to offer blockchain-based design and development solutions in order to build more efficient, fast, secure, and transparent structures in the field of architecture. Blockchain technology will continue to present vast research opportunities on architecture, real estate, banking, and various usage areas. As in all technologies, developments in blockchain technology will be supported and improved by academic studies.

\section{References}

Altunbasak, T. A. (2018). Blok zincir (blockchain) teknolojisi ile vergilendirme. Maliye Dergisi, Ocak-Haziran; 174: 360-371.

Baran, P. (1962). On distributed communications networks. Access date: 05.10.2020. https://ieeexplore.ieee.org/document/1088883/.

Buterin, V. (2014). A next-generation smart contract and decentralized application platform. White Paper.

Carkacioglu, A. (2016). Kripto-Para Bitcoin. Sermaye Piyasası Kurulu Araştırma Dairesi Araştırma $\begin{array}{llll}\text { Raporu, } & \text { ss.42-43. Access } & \text { 05.10.2020. }\end{array}$ http://www.spk.gov.tr/siteapps/yayin/yayingoster/1130.

73 | P a g e

www.iiste.org 
Crosby, M., Nachiappan., Pattanayak, P., Verma, S. and Kalyanaraman V. (2016). Blockchain Technology: Beyond Bitcoin. Applied Innovation Review, Issue:2, 6-19.

Demirel, F. (2017). TÜBİTAK'tan blok zincirine özel araştırma laboratuvarı. Access date: 05.10.2020. https://webrazzi.com/2017/11/21/tubitak-bilgem-blok-zinciri/.

Dilek, Ş. (2018). Blockchain Teknolojisi ve Bitcoin. Siyaset, Ekonomi ve Toplum Araştırmaları Vakfi (SETA), Ankara, Sayı:231, ss.10-30.

Egilmez, M. (2013). Ekonomi Sözlüğü. Access date: 05.10.2020. http://www.mahfiegilmez.com/p/ekonomi-sozlugu.html.

Gunen, E. (2018). İnternetin Yeni Çağı: Blockchain. Fintechtime, Sayı: 8.

Guo, Y. and Liang, C. (2016). Blockchain Application and Outlook in the Banking Industry. Financial Innovation, 2:24, 1-12.

Gupta, V. (2017). A Brief History of Blockchain. Brighton: Harvard Business School Publishing. Access date: 05.10.2020. https://hbr.org/2017/02/a-brief-history-of-blockchain.

HKMA. (2016). Whitepaper On Distributed Ledger Technology. Hong Kong Monetary Authority. Access date: 05.10 .2020 . https://www.hkma.gov.hk/media/eng/doc/key-functions/financialinfrastructure/Whitepaper_On_Distributed_Ledger_Technology.pdf.

Linuma, A. (2018). What Is Blockchain and What Can Businesses Benefit from It? Access date: 05.10.2020. https://www.forbes.com/sites/forbesagencycouncil/2018/04/05/what-is-blockchainand-what-can-businesses-benefit-from-it/\#30d14b8a675f.

Mendi, A. F. and Cabuk, A. (2018). Bitcoin'in Arkasindaki Güç: Blockchain. GSI Journals Serie C: Advancements in Information Sciences and Technologies, 1(1), 12-23.

METI. (2017). Evaluation Forms for Blockchain-Based Systems. Information Economy Division Commerce and Information Policy Bureau, (April 2017), 0-21. Tokyo: Ministry of Economy, Trade and Industry.

Nakamoto, S. (2008). Bitcoin: A peer-to-peer electronic cash system. Access date: 05.10.2020. https://bitcoin.org/bitcoin.pdf.

Pinna, A. and Ruttenberg, W. (2016). Distributed Ledger Technologies in Securites Post-Trading. European Central Bank: Occasional Paper Series. Frankfurt am Main. https://doi.org/10.2866/270533.

Sahin, E. E. (2018). Kripto Para Bitcoin: ARIMA ve Yapay Sinir Ağları ile Fiyat Tahmini, Fiscaoeconomia, Cilt: 2, Sayı: 2.

Savelyev, A. (2017). Contract law 2.0: 'Smart' contracts as the beginning of the end of classic contract law. Information \& Communications Technology Law, 26:2, 116-134.

Turhan, G. (2018). Güncel Akademik Araştırmalar: Sosyal Bilimlere Yönelik Stratejik Bakış Açısı, Blockchain ve Seçim Güvenliği.

Unsal, E. and Kocaoglu, Ö. (2018). Blok Zinciri Teknolojisi: Kullanım Alanları, Açık Noktaları ve Gelecek beklentileri. Avrupa Bilim ve Teknoloji Dergisi, Sayı 13, ss.54-64.

Url-1, Access date: 05.10.2020. http://blokzincir.tubitak.gov.tr/blok-zincir.html. 
Url-2: Access date: 05.10.2020. https://coin-turk.com/sozluk.

Wild, J., Arnold, M. and Stafford, P. (2015). Technology: Banks Seek the Key to Blockchain. Access date: 05.10.2020. https://www.ft.com/content/eb1f8256-7b4b-11e5-a1fe-567b37f80b64.

Yli-Huumo, J., Ko, D., Choi, S., Park, S., Smolander, K. (2016). Where Is Current Research on Blockchain Technology?. A Systematic Review. PloS one, 11(10).

Wilkinson S., Boshevski T., Brandoff J. and Buletin V. (2014). Storj A Decentralized Cloud Storage Network. Access date: 05.10.2020. https://storj.io/storj.pdf.

Xu X., Weber I., Staples M., Zhu L., Bosch J., Bass L., Pautasso C. And Rimba P. (2017) A taxonomy of blockchain-based systems for architecture design. In: 2017 IEEE international conference on software architecture (ICSA), Gothenburg. IEEE, pp 243-252. 